\title{
Compositional Analysis of Isobutylene/ p-Methylstyrene Copolymers by Matrix-Assisted Laser Desorption/Ionization Mass Spectrometry
}

\author{
Frederick J. Cox* and Murray V. Johnston \\ Department of Chemistry and Biochemistry, University of Delaware, Newark, Delaware, USA
}

\author{
Kuangnan Qian and Dennis G. Peiffer \\ ExxonMobil Research and Engineering Company, Annandale, New Jersey, USA
}

\begin{abstract}
A low molecular weight predominantly polyolefin copolymer of isobutylene and para methylstyrene (IMS) was studied using matrix-assisted laser desorption/ionization (MALDI) mass spectrometry. Average composition information derived from the spectra was skewed to higher para methylstyrene (pMS) content as compared to that obtained using multiple NMR techniques, and drifted towards lower pMS incorporation at higher oligomer lengths. Although both observations were initially attributed in total to an inability to ionize the isobutylene component, comparison with subsequent field desorption (FD) mass spectrometry results gave similar values to that obtained via MALDI, even though FD ionizes oligomers not detected by MALDI. Instead, the compositional drift observed with MALDI roughly mirrored the mass distribution, and was determined to arise from a mass bias effect in oligomer ionization and detection. Composition with respect to oligomer mass was found to be relatively constant, although similarly higher in pMS content. Comparison of experimental peaks with a Bernoullian statistical model revealed severe overrepresentation of higher pMS composition oligomers with regard to the calculated distribution. This discrepancy is attributed to preferential ionization of oligomers with greater pMS content, and likely results in the observed difference between MALDI and NMR compositions. (J Am Soc Mass Spectrom 2004, 15, 681-688) @ 2004 American Society for Mass Spectrometry
\end{abstract}

$\mathrm{M}$ ass spectrometry has become a useful method for the compositional analysis of low molecular weight copolymer systems, typically less than $10,000 \mathrm{u}$ [1]. Among the various ionization methods employed, matrix-assisted laser desorption/ionization (MALDI) mass spectrometry is a popular technique, owing to its ability to ionize intact oligomers comprised of a broad range of monomer types by attachment of a metal cation [2-4]. From the distribution of oligomers in the mass spectrum, copolymer composition can usually be calculated either directly from the relative intensities, or by comparison with theoretical statistical oligomer distributions [5-7]. Compared with traditional copolymer composition analysis methods such as NMR or optical spectroscopy, MALDI-MS has the advantage of being able to determine the composition of individual oligomers or seg-

Published online February 27, 2004

Address reprint requests to Dr. M. V. Johnston, Department of Chemistry and Biochemistry, University of Delaware, 107 Brown Laboratory, Newark, DE 19716, USA. E-mail: mvj@udel.edu

${ }^{*}$ Current address: Batelle Eastern Science and Technology Center, 1204 Technology Drive, Aberdeen, MD, 21001-1288, USA. ments of the copolymer distribution without the necessity of an additional separation step. Because of this capability, a single spectrum may be used to characterize the compositional distribution of the oligomers.

However, compositional analysis of a copolymer by MALDI-MS is not always straightforward. A number of reports have suggested that compositional errors may be induced when copolymers with functionally dissimilar monomers are analyzed, as oligomers with different composition may have different ionization efficiencies. For example, silicone copolymers have been reported to have skewed oligomer ionization efficiencies with regard to either hydromethylsiloxane [8] or perfluoroalkyl content [9]. Wilczek-Vera and coworkers found similar skewing with respect to styrene content with $\alpha$-methylstyrene/4-vinylpyridine copolymers [10]. Even with the structurally similar ethylene oxide/ propylene oxide monomers, compositional biases have been observed in copolymer spectra, even though homopolymers of both are easy to ionize [11]. It is reasonable to assume that the MALDI analysis of other copolymers where monomers are even more structurally different would similarly suffer. 
Polyolefin copolymers would seem to be particularly problematic in this regard because of the difficulty in ionizing olefin monomers. Saturated polyolefin homopolymers (e.g., polyethylene, polypropylene, etc.) are notoriously difficult to ionize by MALDI-MS, as there is no relatively polar charge site or unsaturation in the chain at which a metal cation can attach, and attachment to the hydrocarbons themselves has been found to be energetically unfavorable $[12,13]$. Ionization of copolymer oligomers would thus be dependent solely on the non-olefin comonomer. Some researchers have reported limited success in ionizing samples such as polyethylene by MALDI [14], but only at lower mass and with extensive fragmentation. Not surprisingly, scant literature exists for MALDI analysis of copolymers with a high polyolefin content.

A polyolefin copolymer such as isobutylene-co- $p$ methylstyrene (IMS), then, could prove problematic to compositionally analyze by MALDI-MS. Carbocationically copolymerized with a random structure, IMS is commercially important. It offers a number of advantages over both isobutylene homopolymers and other butyl rubber copolymers, such as higher resistance to degradation and enhanced functionalization ability [15]. IMS structure and composition has been previously reported by NMR, but these studies focused on either IMS made by living carbocationic polymerization $[16,17]$, or on the NMR methodology itself [18]. If IMS oligomers could be sufficiently ionized, MALDI-MS could provide additional insight into the copolymerization reaction, and prove to be a valuable alternate characterization method.

Here, we use MALDI-MS to characterize low molecular weight IMS copolymers and extract composition information from spectra that can be obtained. The discrepancy between average composition determined by MALDI and NMR is discussed.

\section{Experimental}

IMS $(\mathrm{Mn}=1240,2400$, and $5000 \mathrm{~g} / \mathrm{mol})$ copolymers containing para methylstyrene compositions 13,10 , and $12 \%$ respectively as determined by NMR, and homopolymers of isobutylene $(\mathrm{Mn}=2900 \mathrm{~g} / \mathrm{mol})$ and para methylstyrene $(\mathrm{Mn}=2400$ and $5200 \mathrm{~g} / \mathrm{mol})$, were obtained from ExxonMobil Research and Engineering (Annandale, NJ). Indoleacrylic acid (IAA), all trans retinoic acid (RA), silver trifluoroacetate (AgTFA), and copper (II) trifluoroacetate (Cu (II)TFA), and polyethylene glycol (PEG) were obtained from Aldrich (Milwaukee, WS) and used without further purification. All solutions were prepared in tetrahydrofuran or toluene at a concentration of $10 \mathrm{mg} / \mathrm{mL}$; matrix solutions were prepared fresh weekly. Matrix, cation, and analyte were mixed in a volume ratio of 8:1:4 respectively. A $1 \mathrm{uL}$ aliquot of the mixture was applied to the probe and allowed to air dry. Because tetrahydrofuran is highly volatile, the sample spots tended to spread over a wide area of the sample plate and resemble those produced by spray techniques. Calibration was performed with PEG of varying molecular weights in the case of the pMS homopolymers and with pMS for the IB-pMS copolymers.

Mass spectra were acquired on a Bruker (Billerica, MA) Biflex III MALDI time-of-flight mass spectrometer operating in reflectron mode using delayed extraction with the Scout 384 source. Pulsed ion deflection was used to enhance the analyte signal by avoiding matrix signal saturation and desensitization. Spectra were acquired with a minimum of 50 shots per spectrum for the homopolymer to approximately 3000 shots for the copolymer spectra. The probe position was moved approximately every 5-10 shots when visible sample depletion was observed and/or when the signal decreased significantly.

MALDI data analysis was performed using the Polymerix software package from Sierra Analytics (Modesto, CA). Using this software, spectra were first baseline corrected, centroided, and deisotoped, and the resultant peaks identified by matching to theoretical masses of possible oligomers. The impact of peak picking parameters included in the software package was minimal. Centroided peaks were separated generally by $1 \mathrm{~m} / \mathrm{z}$ or more, and the mass tolerance to match the software generated oligomer masses to the observed masses was less than 1 . Peak areas rather than peak heights were used in the analysis because the mass resolving power changed significantly over the mass range studied. Calculations of composition and modeling were performed using the Excel spreadsheet software from Microsoft (Redmond, WA) on peaks areas exported from Polymerix.

Field Desorption Mass Spectrometry (FDMS) was conducted on a VG-ZAB mass spectrometer. About 10 $\mathrm{mg}$ of the polymer samples were dissolved in $10 \mathrm{ml}$ of toluene. One $\mu \mathrm{l}$ of the solution was directly deposited onto the FD emitter using a syringe. Molecules were ionized by an intense electric field $\left(10^{7}-10^{8} \mathrm{~V} / \mathrm{cm}\right)$ applied between carbon dendrimers on a thin filament and the source electrode. Electrons were removed from the analyte molecule via a process known as the quantum tunneling effect. The emitter was heated with a ramping current from 0 to $65 \mathrm{~mA}$ to assist the desorption of the polymer molecules. The extraction electrodes were heated with 1.2 A current (corresponding to about $225^{\circ} \mathrm{C}$ ) to avoid analyte condensation. This process generates intact polymer molecular ions with minimal fragmentation.

\section{Results and Discussion}

\section{Copolymer and Homopolymer Spectra}

Homopolymers consisting of each of the comonomers of IMS were first examined to determine their respective ionization properties. For the polyisobutylene sample, no MALDI signal was observed over a wide mass range when analyzed in the above fashion other than 


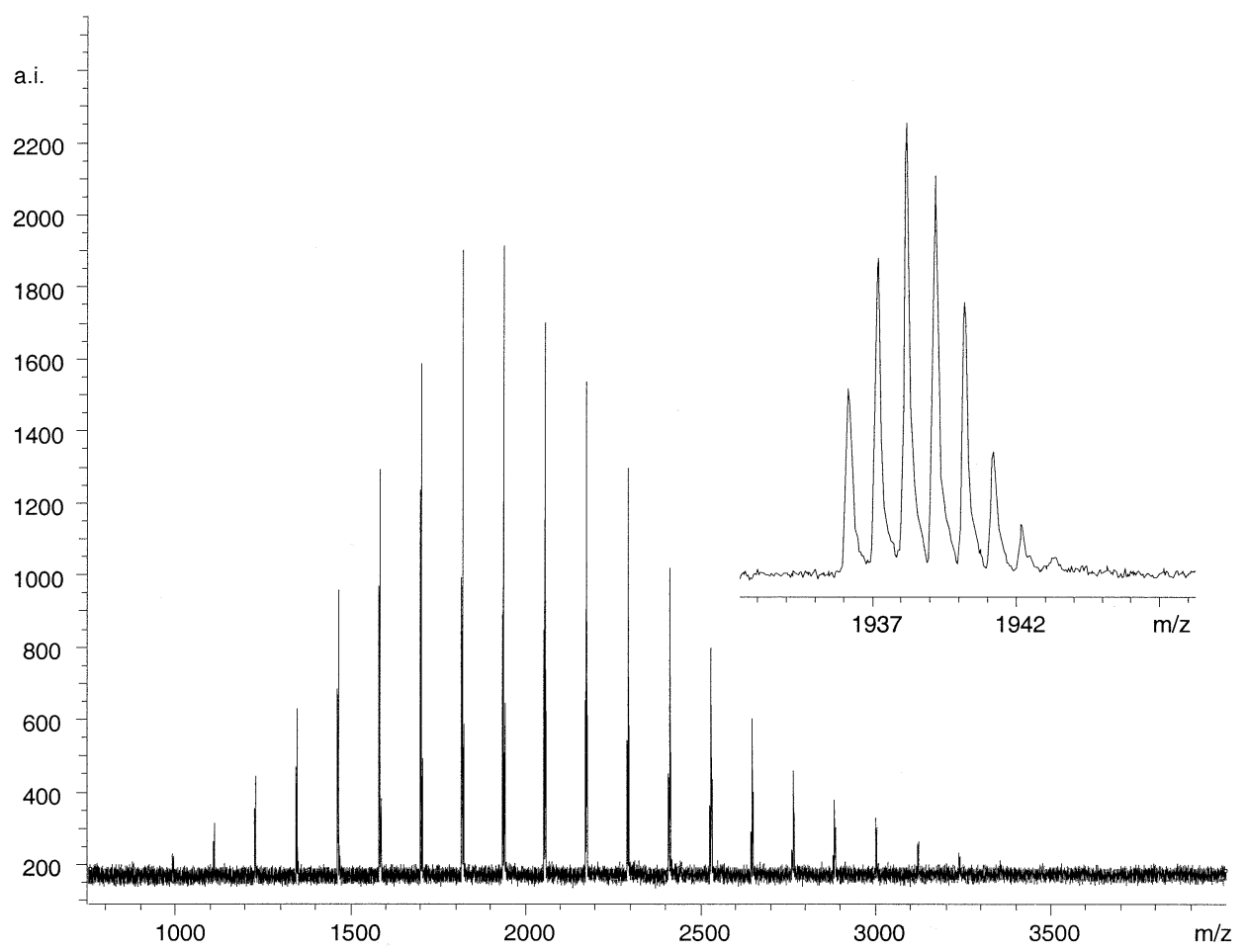

Figure 1. MALDI-MS spectrum of pMS homopolymer. Inset shows isotopic resolution of peaks at $1925 \mathrm{~m} / \mathrm{z}$.

ions attributable to matrix and silver clusters. This was expected because of the lack of a suitable ionization site on the non-polar isobutylene monomer.

Both the pMS 2400 and pMS 5200 homopolymer spectra were obtained with ease using silver cationization. Figure 1 shows the MALDI mass spectrum of the pMS 2400 homopolymer, giving continuous oligomer signals from just above 900 to $3500 \mathrm{~m} / \mathrm{z}$. Isotopic distributions corresponding to the silver cationized oligomers are separated by $118 \mathrm{~m} / \mathrm{z}$, equivalent to the mass of a pMS monomer. When the copper TFA salt is used in place of silver, analysis of the pMS homopolymer gives similar results. In that case, the respective oligomer masses are shifted downward from the silver cationized spectra by $44 \mathrm{~m} / \mathrm{z}$ due to the binding of a copper cation $\left({ }^{63} \mathrm{Cu}\right)$ rather than silver $\left({ }^{107} \mathrm{Ag}\right)$.

In comparison to the pMS homopolymer, spectra of high signal-to-noise ratio obtained for the IMS copolymers required both more shots and relatively higher laser powers. Figure 2 shows a spectrum of IMS 1240 obtained with silver cationization and dithranol. Other matrices (RA and IAA) were investigated but offered no significant advantage over dithranol for the IMS copolymers. Copolymer oligomer peaks are visible from 600 to over $1800 \mathrm{~m} / \mathrm{z}$. The wide polydispersity of the copolymer $(\sim 2)$ is notable. At lower $m / z$, individual isotopic peaks are easily resolved, but they could not be resolved at $\mathrm{m} / \mathrm{z}$ values greater than 1500 . No molecular weight calculation was performed because of the inability to properly quantify these peaks.

Examination of the spectrum in Figure 2 reveals periodic groups of peaks (Figure 3). The groups are separated by $56 \mathrm{~m} / \mathrm{z}$, which corresponds to the addition of an isobutyl group, while each isotopic distribution (arising from the silver 107/109 and carbon 12/13 isotopes) in the cluster is separated by $6 \mathrm{~m} / \mathrm{z}$. This is accounted for by the mass difference between one para methylstyrene unit $(118 \mathrm{u})$ and two isobutyl units (2.56 $=112 \mathrm{u}$ ). Thus, moving from left to right in an oligomer group, a gain of $6 \mathrm{~m} / \mathrm{z}$ corresponds to one more para methylstyrene monomer and two less isobutyl mono-

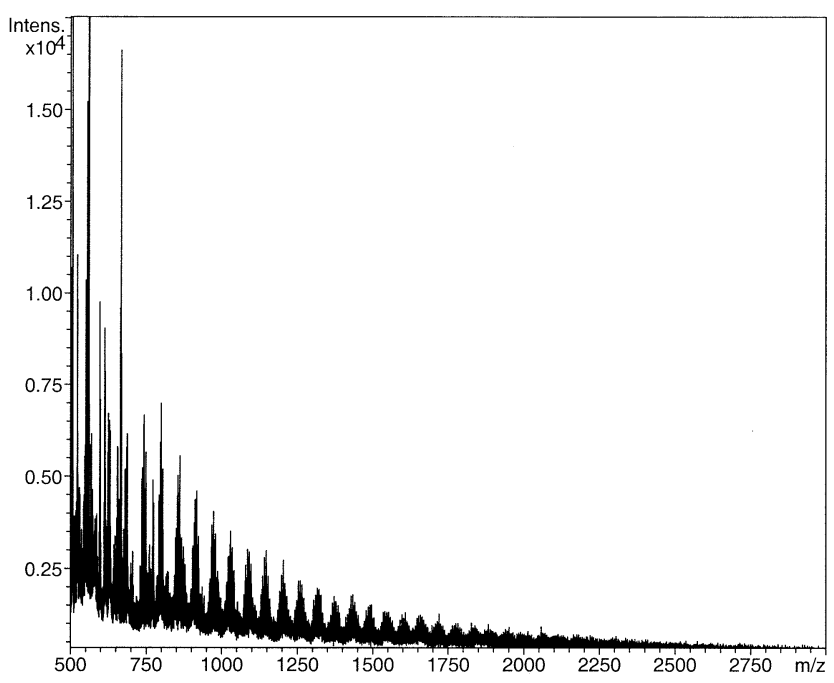

Figure 2. Spectrum of IMS 1240. 


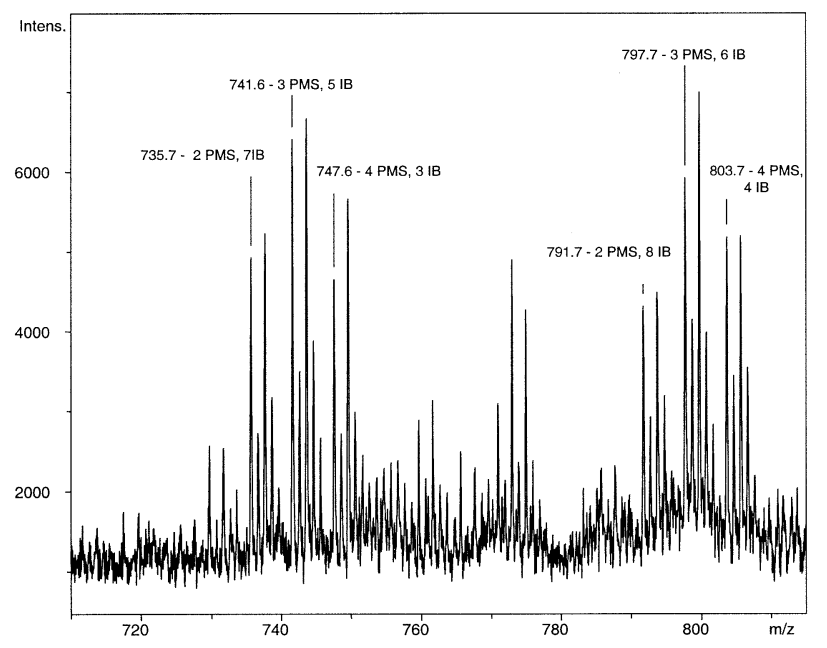

Figure 3. Expanded view of spectrum in Figure 2 showing oligomer identities in two groupings of peaks. The number of units of para methylstyrene (PMS) and isobutylene (IB) are indicated for the first peak in each isotopic distribution.

mers. When moving from one grouping of oligomers to another, one isobutyl group is gained or lost. In oligomer groups, oligomer peaks with one pMS group were generally negligible in comparison to groups containing two or more such groups, and those with zero pMS (i.e., isobutylene homopolymer) were nonexistent. At lower $\mathrm{m} / \mathrm{z}$ values, no oligomers with greater than 4 pMS units were observed, but oligomers with 5 , 6 , and higher numbers of pMS monomers were observed above $900 \mathrm{~m} / \mathrm{z}$. This is likely due to the limited number of combinations of pMS and IB monomers at lower $\mathrm{m} / \mathrm{z}$ values for a $13 \%$ pMS composition rather than an ionization bias.

It is interesting to note that the peaks observed indicate the presence of unsaturated end groups (either a pMS or an IB group in the oligomer is unsaturated, containing one double bond). For example, the $\sim 741$ $\mathrm{m} / \mathrm{z}$ peak corresponds to a combination of $3 \mathrm{pMS}$ and 5 IB monomer units, with a single ${ }^{107} \mathrm{Ag}$ cation and one unsaturation site. Although ions like this are predominant in the MALDI spectrum, NMR data for the IMS 1240 copolymer indicates that there is less than 1 olefin per 5000 carbons. While this difference has not been reconciled, it is plausible that the intensities of unsaturated oligomers are greatly enhanced due to favorable ionization efficiencies.

No signal could be obtained for the IMS 1240 copolymer using CuTFA as the cationization agent. This is in stark contrast to the ease with which spectra were obtained with the pMS homopolymer using both copper and silver cationization. This suggests that copper cationization may occur by a different route than silver cationization. Copper binding may require closed spacing of aromatic rings than is present in this composition copolymer.

Attempts to obtain MALDI spectra of higher mass IMS copolymers with silver cationization were unsuc- cessful. Though unresolved undulations (which may be oligomer groupings) appear in the baseline of the spectrum of IMS 2400, no oligomer peaks could be resolved, and positive identification could not be made. Increasing laser power to increase signal intensity resulted only in increasing silver clusters and other unidentified interferences. Spectra for IMS 5000 were similar, with no oligomer peaks observed. The inability to view higher mass copolymer oligomers is perhaps not surprising in light of the decreased detection efficiency of MALDI-MS for higher molecular weight oligomers in samples of high $(>1.4)$ polydispersity [19, 20]. MALDI spectra were obtained, however, by separating the higher molecular weight samples into lower polydispersity fractions by GPC (data not shown).

\section{Composition Analysis}

Because of the inability to detect oligomers in unfractionated IMS 2400 and 5000 samples, compositional analysis was limited to IMS 1240. Average composition was determined directly from 6- to 18-mer oligomer distributions in the copolymer spectrum. Eq 1 was used to calculate the percent composition from the MALDI spectrum:

$$
\text { Mol \% pMS }=\frac{\sum_{n=6}^{18}\left[\sum_{x=0}^{n}\left(M S_{x} I_{(n-x)}\right)(x / n)\right]}{\sum_{n=6}^{18}\left[\sum_{x=0}^{n}\left(M S_{x} I_{(n-x)}\right)\right]}
$$

where $n$ refers to the number of monomers and $\mathrm{MS}_{\mathrm{x}} \mathrm{I}_{(n-\mathrm{x})}$ is the sum of the areas of all the isotopic peaks of the oligomer containing $x$ para methylstyrene monomers and $(n-x)$ isobutyl monomers. Out of all possible oligomer peaks in the above chain length range, only those with sufficient signal-to-noise ratio (greater than 2:1) identified as copolymer peaks by the Polymerix software were used in the equation. This usually included peaks from 600 to $1300 \mathrm{~m} / \mathrm{z}$, with the range varying slightly depending on the specific spectrum. The para methylstyrene mole percentage as determined from above was calculated for three spectra, and found to be $36 \pm 1$, nearly three times the value obtained through NMR (13\%). Because the composition calculation is based on the relative ratios of the oligomer peaks of various compositions in the spectra, the discrepancy between the MALDI and NMR values is by necessity caused by an overrepresentation of oligomers with high pMS content (or, conversely, an under representation of oligomers with high IB content).

This oligomer skewing may be explained by a number of factors. First, as discussed earlier, the ionization of a copolymer oligomer may depend on composition. Because isobutylene monomers likely do not ionize, oligomers containing more styrene and less isobutylene content would have a higher probability of ionization, 


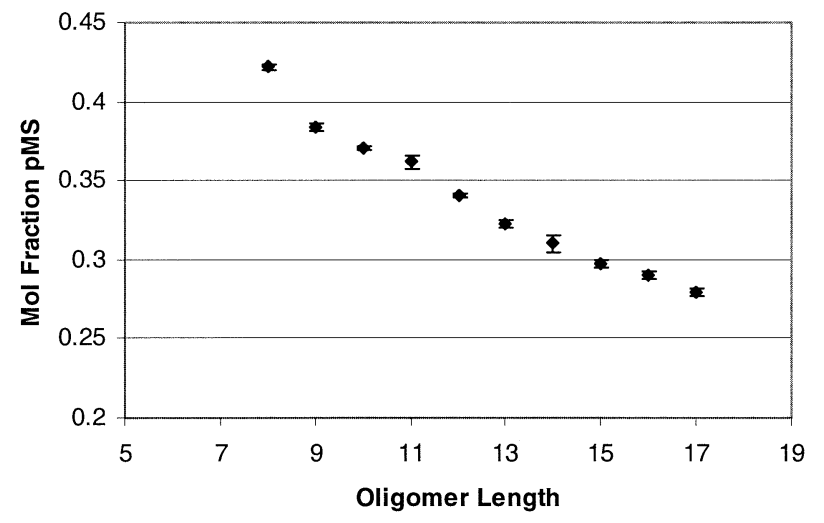

Figure 4. Plot of average oligomer composition versus oligomer length with the instrument optimized for performance below 1000 $\mathrm{m} / \mathrm{z}$. Error bars represent one S.D. from the mean.

and thus would increase the apparent composition. Another explanation may lie in the polydispersity of the copolymer and the relatively large difference in mass between para methylstyrene $(118 \mathrm{u})$ and isobutylene (56 u) monomers. However, if the high polydispersity of the copolymer causes an ionization and detection bias towards oligomers of high molecular weight, one would expect oligomers of greater pMS content to be discriminated against, as they would have increased weight relative to oligomers of the same length but greater IB content. If this were the sole reason, the composition determined by MALDI-MS would be lower rather than higher. Of course, the effects of polydispersity may still be represented in the value obtained, and removing these effects would increase the discrepancy between the MALDI and NMR values even more. Finally, the copolymer may also have compositional heterogeneity. If oligomers with smaller chain length are richer in pMS monomers (for instance, because of different reaction rates for addition of the two monomers), and the polydispersity prevents detection of longer chains which are predominantly IB, the apparent bulk composition would appear also richer in pMS.

An important feature of mass spectrometry in copolymer analysis is the ability to evaluate changes in comonomer composition as a function of chain length. In order to examine the various possibilities further, the composition at each oligomer length was determined using eq 2:

Mol fraction pMS of n-oligomer

$$
=\frac{\sum_{x=0}^{n}\left(M S_{x} I_{(n-x)}\right)(x / n)}{\sum_{x=0}^{n}\left(M S_{x} I_{(n-x)}\right)}
$$

Figure 4 shows the plot of oligomer composition as a function of oligomer length. pMS content appears to decrease linearly with increasing oligomer length, and, at first glance, would seem to implicate compositional heterogeneity. This may be the case if an initial block of pMS is formed, followed by subsequent addition, in which case smaller oligomers have a higher proportion of the block to rest of the oligomer. Alternately, if the reactivity ratios of the monomers are different and one of the monomer feeds is exhausted at high conversion, composition can change with increasing chain length. However, in the case of IMS copolymerization there is random incorporation of the comonomers and the reactivity ratios are nearly identical [15]. True compositional heterogeneity arising from the sample is thus unlikely.

Oligomer ionization efficiencies dependent on the composition or absolute number of PMS monomers could also explain the drift. If ionization of an oligomer required a certain threshold number of styrene ringsfor example, two for pi-stacking with silver cations [21]-larger oligomers would be easier to observe, as oligomers with greater length but constant composition have a higher likelihood of having more PMS monomers than smaller oligomers. To determine whether this was truly the case, IMS 1240 was examined by field desorption mass spectrometry (FD-MS). FD has the capability to ionize intact saturated hydrocarbon oligomers similar to polyisobutylene not amenable to MALDI, in addition to being able to easily analyze polystyrenes [22, 23]. In the case of IMS 1240, though, a similar decrease in pMS content with increasing oligomer length was observed even though the overall composition determined from the spectrum, $25 \%$, was closer to that obtained by NMR, $13 \%$. Subsequent analysis of equimolar mixtures of IMS with polyisobutylene homopolymer of similar molecular weight revealed that although FD could ionize oligomers that MALDI could not, the ionization efficiency of oligomers with at least some styrene content was higher than those with only isobutylene, as spectra were dominated by the copolymer. This is consistent with the observation that the FD ionization efficiency of aromatics is higher than that of saturated hydrocarbons.

Still uncertain of the explanation for the observed drift in composition, we noted that the pattern observed in Figure 4 is similar to the mass distribution of the copolymer in Figure 2. This suggests that the inability to detect higher mass oligomers-a result of the high polydispersity-causes the decreasing composition because of the significant mass difference between the monomers. Oligomers with more isobutylene, being lower in mass, would therefore be favored. If instrumental detection facilitated this curve, then enhancement of the ability to detect higher mass oligomers should eliminate the compositional drift. In the case of Figure 4, the mass spectrometer was set to a "short" delayed extraction time and voltages optimized for resolution and detection below $1000 \mathrm{~m} / \mathrm{z}$. However, for higher masses, it is known that a longer delay, "me- 


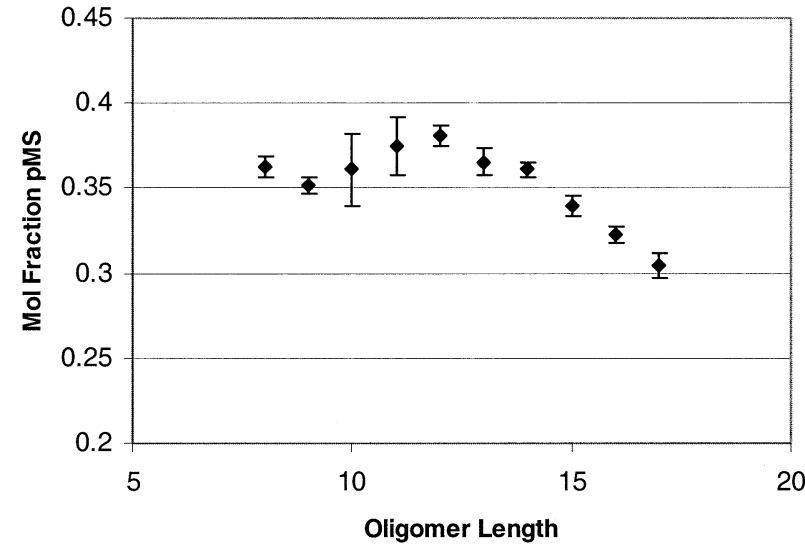

Figure 5. Plot of oligomer composition versus oligomer length, but with instrument optimized for performance at $1600 \mathrm{~m} / \mathrm{z}$. Note the smaller oligomer length dependence compared with Figure 4. Error bars represent one S.D. from the mean.

dium", often works better, and different voltage values must be used [24]. Figure 5 is the result of a similar compositional analysis as performed in Figure 4 but using instrumental parameters optimized for detection at $1600 \mathrm{~m} / \mathrm{z}$, with medium delay. Clearly, different measurement parameters cause different values of pMS content to be obtained as the oligomer length dependence is removed. Therefore, the observed compositional drift in Figure 4 is likely caused by the poor ability of MALDI-TOF to resolve and detect higher mass oligomers in polydisperse samples.

Further evidence for this can be gained by looking at composition as a function of oligomer mass rather than length. The composition of each cluster of oligomers of similar $\mathrm{m} / \mathrm{z}$ seen in Figure 2 was calculated in the following manner:

Oligomer grouping composition

$$
=\frac{\sum_{x=0}^{n}\left(M S_{x} I_{(n-2 x)}\right)(x / n)}{\sum_{x=0}^{n}\left(M S_{x} I_{(n-2 x)}\right)}
$$

Figure 6 shows this composition as a function of the oligomer length of the theoretically lowest mass oligomer in each grouping, which would be an oligomer with all isobutylene (if it were detectable). Plots representing both sets of instrumental parameters, optimized for $\mathrm{m} / \mathrm{z}$ values below 1000 and at 1600 as discussed above, are shown. The good agreement between the two plots indicates that the broad mass range over which oligomers of any given length may be detected and the variable detection efficiency of those oligomers at different masses are responsible for the curves in Figures 4 and 5. The shape of the composition curve in Figure 6 is attributed to the "true" distribution of the copolymer. If the total amount of oligomers at each

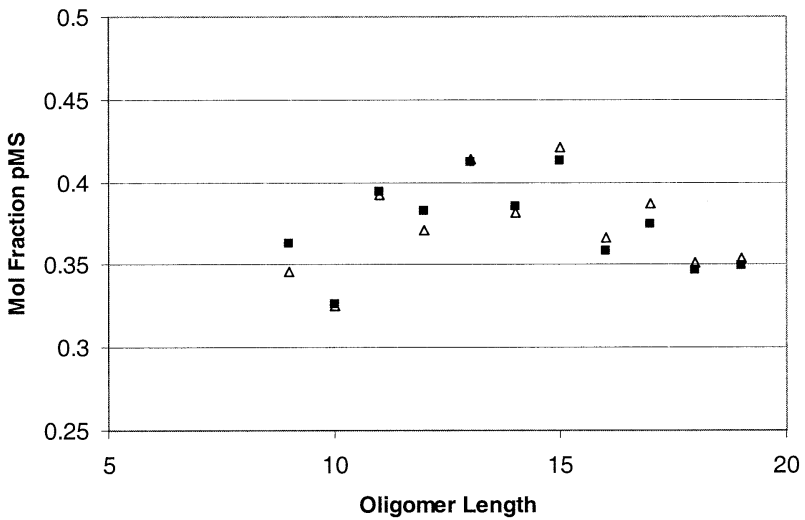

Figure 6. Plot of composition versus oligomer mass for same spectra analyzed in Figures 4 and 5. Each set of data points (squares from Figure 4 data, triangles from Figure 5 data) represents average composition of oligomer groupings versus oligomer length of lowest mass peak in each oligomer grouping from spectra obtained with the instrument optimized at different masses. These plots are nearly indistinguishable for the two sets of instrumental parameters.

length was the same, assuming a random copolymerization, the composition of each peak grouping should be the same, as each peak grouping represents a distributed sampling of the peaks from various oligomer lengths. Different total amounts of oligomers at each length skew the compositions for each grouping.

Although differences in detection at different $\mathrm{m} / \mathrm{z}$ values may explain the compositional curve observed, it does not explain the large discrepancy in composition between MALDI and NMR. It is worth noting that in all of the above methods for examining composition as a function of length or mass, and changes in instrumental parameters, the overall composition remained the same, roughly $36 \mathrm{~mol} \%$ pMS. Exhausting all other possibilities, it seems likely that this general overrepresentation of pMS content is due to decreased ionization efficiency of oligomers with smaller pMS to IB ratio. To determine the extent of this effect, experimental distributions of oligomers were compared to theoretical distributions generated using a Bernoullian chain statistics. Bernoullian statistics are generally assumed when the addition of either of the comonomers is dependent only on the composition of that monomer in the feed, rather than the identity of the chain end, and are a good assumption for a random copolymer such as IMS [25, 26]. From the molar fraction pMS of the sample as determined by NMR $(X)$, and the number of monomers of each type $(m, n)$ in the oligomer, the intensities of oligomers at each length $\left(\mathrm{I}_{\mathrm{MS} I n}\right)$ can be calculated by the following:

$$
I_{M S_{m} I_{n}}=\frac{(m+n) !}{m ! n !}[X]^{m}[1-X]^{n}
$$

A comparison between a theoretical $13 \mathrm{~mol} \%$ pMS distribution and experimental data is shown in Figure 7 for the oligomer grouping beginning with a 12-mer at 


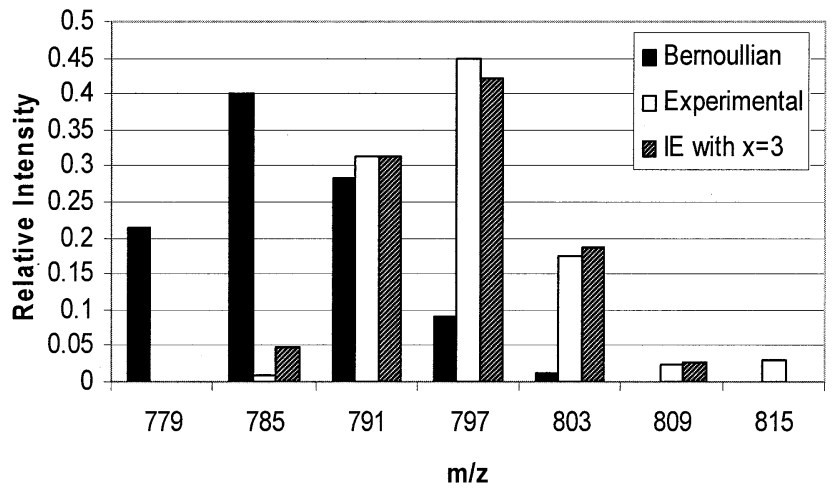

Figure 7. Comparison of experimental peak distribution, calculated distribution, and the calculated distribution using the IE term.

$779 \mathrm{~m} / \mathrm{z}$ (all IB) and ending with a 6-mer at $815 \mathrm{~m} / \mathrm{z}$ (all pMS). The Bernoullian plot assumes that oligomer lengths 6-12 each contribute equally to mass spectrum. Although not strictly valid for a distribution of oligomers, we use this approach because the error induced by this assumption for a highly polydisperse copolymer is likely less than any error that would be introduced by looking at a single oligomer length with peaks over a wide mass range. Comparison of Figures 4 and 5 with Figure 6 shows less variation in composition between oligomer groupings than oligomer length, and the overall curve is flatter in Figure 6 for either of the data sets. Nonetheless, the variation between the Bernoullian model distribution and experimental is great. Recall that in an oligomer grouping, an increase of $6 \mathrm{~m} / \mathrm{z}$ results in the addition of a single pMS at the expense of two IB. Therefore, as compared to the model, oligomers with one or less pMS monomers are severely underrepresented in the experimental data, while oligomers with greater pMS content are over-represented. The agreement factor, a measure of the fit of the model distribution to the experimental, was 1.2, which is relatively poor. A situation in which MALDI of oligomers is controlled by pMS content would seem to be the most likely explanation.

To account for this difference in copolymer oligomer ionization, Koster and coworkers proposed the introduction of a relative ionization efficiency term, IE, in the calculation of the theoretical distribution when using chain statistics [27]. In this case, the calculation of theoretical oligomer intensities by Bernoullian statistics would need to be modified so that the intensity of a particular oligomer is not only determined by the mole fraction of the sample and the relative amounts of monomers, but is also dependent on the ionization efficiency of that oligomer. Eq 4 becomes:

$$
I_{M S_{m} I_{n}}=I E_{\left(M S_{m} I_{n}\right.} \frac{(m+n) !}{m ! n !}[X]^{m}[1-X]^{n}
$$

Although no formula or value for such a term is determined in that work, Servaty and coworkers suggest a composition dependent ionization probability that increased linearly with composition could account for the difference, albeit with copolymers dissimilar to those studied here [8]. We first used the linear assumption to adjust the theoretical distribution by multiplying the probabilities for each oligomer by the fractional composition of pMS in each oligomer, so that the IE term in eq 5 becomes equal to $\mathrm{m} /(\mathrm{m}+\mathrm{n})$. Although the agreement factor was improved (0.78), it was still relatively high. Through repeated trial and error, we discovered that a higher-order estimate for ionization efficiency, $[m /(m+n)]^{x}$, had to be used to obtain a good fit with the experimental data (Figure 7). Using the cube of the pMS fraction in the oligomers $(x=3)$ in the ionization efficiency term resulted in a good agreement factor of 0.14 . This suggests that the amount of pMS in the oligomers strongly controls desorption/ ionization by MALDI. Furthermore, because the dependence is not linear, groupings of pMS monomers in close proximity on the chain seem to be more favorable for ionization, as such groupings would be exponentially higher at greater pMS content.

\section{Conclusions}

MALDI mass spectra can be obtained for IMS copolymers, but only at relatively low molecular weights. Spectra could not be obtained at $\mathrm{Mn}=2400$ and above, likely due to the wide polydispersity of the copolymers. In the spectra that were obtained, the monomer composition was found to deviate greatly from that obtained by NMR. A strong inverse relationship was also observed between oligomer length and composition. This relationship was found to be an artifact of MALDITOF mass discrimination effects, as the trend could be eliminated with changes in instrumental parameters. This relationship was not observed when determining comonomer composition as a function of oligomer mass rather than length. Comparison of experimental oligomer distributions to a Bernoullian statistical model revealed severe overrepresentation of oligomers with greater relative amounts of pMS. However, good agreement between the model and experimental data could be obtained by introduction of an ionization efficiency term to the Bernoullian model. Facile ionization of pMS rich oligomers appears to cause the composition discrepancy between MALDI and NMR.

\section{Acknowledgments}

This research was supported under NSF grant CHE-9634238. The research was also in part supported by ExxonMobil Research and Engineering Company. FC acknowledges support from a University of Delaware Competitive Graduate Fellowship. The authors acknowledge Scott Campbell (Sierra Analytics) for use and assistance with the Polymerix software. 


\section{References}

1. Montaudo, M. S. Mass Spectra of Copolymers. Mass Spectrom. Rev. 2002, 21, 108-144.

2. Rader, H. J.; Schrepp, W. MALDI-TOF Mass Spectrometry in the Analysis of Synthetic Polymers. Acta Polym. 1998, 49(6), 272-293.

3. Nielen, M. W. F. MALDI Time-of-Flight Mass Spectrometry of Synthetic Polymers. Mass Spectrom. Rev. 1999, 18, 309-344.

4. Hanton, S. D. Mass Spectrometry of Polymers and Polymer Surfaces. Chem. Rev. 2001, 101, 527-569.

5. Montaudo, G.; Montaudo, M. S. Polymer Characterization Methods. In Mass Spectrometry of Polymers; Montaudo, G.; Lattimer, R. P., Eds.; CRC Press: Boca Raton, FL, 2002; pp 41-112.

6. Montaudo, G.; Garozza, D.; Montaudo, M. S.; Puglisi, C.; Samperi, F. Molecular and Structural Characterization of Polydisperse Polymers and Copolymers by Combining MALDI-TOF Mass Spectrometry with GPC Fractionation. Macromolecules 1995, 28, 7983-7989.

7. Wilczek-Vera, G.; Danis, P. O.; Eisenberg, A. Individual Block Length Distributions of Block Copolymers of Polystyreneblock-Poly( $\alpha$-methylstyrene). Macromolecules 1996, 29, $4036-$ 4044.

8. Servaty, S.; Kohler, W.; Meyer, W. H.; Rosenauer, C.; Spickerman, J.; Rader, H. J.; Wegner, G.; Weier, A. MALDI-TOF-MS Copolymer Analysis: Characterization of a Poly(dimethylsiloxane)-co-Poly(hydromethylsiloxane) as a Precursor of a Functionalized Silicone Graft Copolymer. Macromolecules 1998, 31, 2468-2474.

9. Yoshida, S.; Yamamoto, S.; Takamatsu, T. Detailed Structural Characterization of Modified Silicone Copolymers by MatrixAssisted Laser Desorption/Ionization Time-of-Flight Mass Spectrometry. Rapid Commun. Mass Spectrom. 1998, 12, 535544.

10. Wilczek-Vera, G.; Yu, Y.; Waddell, K.; Danis, P. O.; Eisenberg, A. Detailed Structural Analysis of Diblock Copolymers by Matrix-Aassisted Laser Desorption/Ionization Time-of-Flight Mass Spectrometry. Rapid Commun. Mass Spectrom. 1999, 13, 764-777.

11. Chen, R.; Zhang, N.; Tseng, A. M.; Li, L. Effects of MatrixAssisted Laser Desorption/Ionization Experimental Conditions on Quantitative Compositional Analysis of Ethylene Oxide/Propylene Oxide Copolymers. Rapid Commun. Mass Spectrom. 2000, 14, 2175-2181.

12. Reinhold, M.; Meier, R. J.; Koster, C. G. How Feasible is Matrix-Assisted Laser Desorption/Ionization Time-of-Flight Mass Spectrometry Analysis of Polyolefins? Rapid Commun. Mass Spectrom. 1998, 12, 1962-1966.

13. Ehlers, A. W.; Koster, C. G.; Meier, R. J.; Lammertsma, K. MALDI-TOF-MS of Saturated Polyolefins by Coordination of Metal Cations: A Theoretical Study. J. Phys. Chem. A 2001, 105, 8691-8695.
14. Chen, R.; Yalcin, T.; Dai, Y.; Wallace, W. E.; Guttman, C. M.; Li, L. Laser Desorption Ionization and MALDI Time-of-Flight Mass Spectrometry for Low Molecular Mass Polyethylene Analysis. J. Am. Soc. Mass Spectrom. 2001, 12, 1186-1192.

15. Wang, H. C.; Powers, K. W. Functionalized p-Methylstyrene/ Isobutylene Copolymers Offer Wide Eange of Properties. Elastomerics 1992, 124, 14-19.

16. Lubnin, A. V.; Orszagh, I.; Kennedy, J. P. The Microstructure of Poly(isobutylene-co-p-methylstyrene) by NMR Spectroscopy. J. M. S. Pure Appl. Chem. 1995, 11, 1809-1830.

17. Orszagh, I.; Nagy, A.; Kennedy, J. P. Living Carbocationic Copolymerizations. I. Synthesis and Characterization of Isobutylene/p-Methylstyrene Copolymers. J. Phys. Org. Chem. $1995,8,258-272$.

18. Ashbaugh, J. R.; Ruff, C. R.; Shaffer, T. D. Characterization of Signals in the Solution 1H NMR Spectrum of Poly(isobutylene-co-p-methylstyrene) and Their Utility. J. Polym. Sci. A Polym. Chem. 2000, 38, 1680-1686.

19. McEwen, C. N.; Jackson, C.; Larsen, B. S. Instrumental Effects in the Analysis of Polymers of Wide Polydispersity by MALDI Mass Spectrometry. Int. J. Mass Spectrom. Ion Processes 1997, 160, 387-394.

20. Schriemer, D. C.; Li, L. Mass Discrimination in the Analysis of Polydisperse Polymers by MALDI Time-of-Flight Mass Spectrometry. 2. Instrumental Issues. Anal. Chem. 1997, 69, 41764183.

21. Gidden, J.; Wyttenbach, T.; Batka, J. J.; Weis, P.; Bowers, M. T.; Jackson, A. T.; Scrivens, J. H. Poly(ethylene terephthalate) Oligomers Cationized by Alkali Ions: Structures, Energetics, and Their Effect on Mass Spectra and the Matrix-Assisted Laser Desorption/Ionization Process. J. Am. Soc. Mass Spectrom. 1999, 10, 883.

22. Lattimer, R. P.; Schulten, H. R. Field Desorption of Hydrocarbon Polymers. Int. J. Mass Spectrom. Ion Phys. 1983, 52, 305-116.

23. Evans, W. J.; DeCoster, D. M.; Greaves, J. Evaluation of Field Desorption Mass Spectrometry for the Analysis of Polyethylene. J. Am. Soc. Mass Spectrom. 1996, 7, 1070-1074.

24. Service and Maintenance Manual, FLEX III V. 0.2; Bruker Saxonia Analytik GmBH: Leipzig, Germany, 2000, pp 28-34.

25. Montaudo, M.; Ballistreri, A.; Montaudo, G. Determination of Microstructure in Copolymers. Statistical Modeling and Computer Simulation of Mass Spectra. Macromolecules 1991, 24 , 5051-5057.

26. Montaudo, M.; Montaudo, G. Further Studies on the Composition and Microstructure of Copolymers by Statistical Modeling of Their Mass Spectra. Macromolecules 1992, 25, 42644280.

27. Koster, S.; Mulder, B.; Duursma, M. C.; Boon, J. J.; Philipsen, H. J. A; Velde, J. W.; Nielen, M. W. F.; de Koster, C. G.; Heeren, R. M. A. Quantitative Analysis of Copolymers: Influence of the Structure of the Monomer on the Ionization Efficiency in Electrospray Ionization FTMS. Macromolecules 2002, 35, 49194928. 